\title{
Types of Generalized Open Sets with Ideal
}

\author{
Rodyna A. Hosny \\ (1)Department of Mathematics, \\ Faculty of Science, \\ Zagazig University, Egypt \\ ${ }^{(2)}$ Department of Mathematics and Statistics, Faculty \\ of Science, Taif University, KSA
}

\author{
Deena Al-Kadi \\ Department of Mathematics and Statistics, Faculty \\ of Science,Taif University, KSA
}

\begin{abstract}
The aim of this paper is to introduce and study the notions of $\alpha$ (resp., pre, semi, $\beta$ )-open sets in terms of an ideal I. These concepts generalize the usual notions of $\alpha$ (resp., pre, semi, $\beta$ ) open sets. Also, several of their topological properties are investigated.
\end{abstract}

\section{Keywords}

Pre-open set, Semi-open set, $\alpha$-open set, $\beta$-open set, Topological ideal, I-pre-open set, I-semi-open set.

\section{INTRODUCTION}

It is well-known that a large number of papers is devoted to study classes of subsets of a topological space, containing the class of open sets, and possessing properties more or less similar to those of open sets e.g. pre-open sets [7, 10, 11], semi-open sets [9], $\alpha$-open sets [13] and $\beta$-open sets [1] (called semi-preopen sets [2]).

Relevant to the present work, the idea of using topological ideals in describing topological notions, which for some years now has been an interesting subject for investigation [see some of the pioneering works in 3, 4, 5]. We recall here that an ideal $\mathrm{I}$ on a topological space $(\mathrm{X}, \tau)$ is a non-empty collection of subsets of $\mathrm{X}$ having the heredity property (that is, if $A \in I$ and $B \subseteq A$, then $B \in I$ ) and also satisfying finite additively (that is, if $A, B \in I$, then $A \cup B \in I$ ).

The concept of I-semi-open and I-pre-open sets in ideal topological spaces were introduced and studied by [12] and [14], respectively. These definitions turned our attention to proceed in this direction and introduce the concept of $\alpha$ (resp., $\beta$ )-open sets with respect to an ideal I, which is said to be I- $\alpha$ (resp., I- $\beta$ )-open sets. These notations are extensions of usual concepts $\alpha$ (resp., $\beta$ )-open sets. Some of their basic properties will be obtained. These new definitions enable us to establish relationships between definitions of I-semi-open, I-pre-open sets, I- $\alpha$-open sets and I- $\beta$-open sets.

Throughout the current work a topological space $(X, \tau)$ (or simply $\mathrm{X}$ ) will be utilized, where no separation axioms are assumed. The usual notations $\mathrm{Cl}$ (A) for the closure and Int (A) for the interior of a subset $\mathrm{A}$ of a topological space $(\mathrm{X}, \tau)$ is used.

We recall some notions which are used in our sequel.

Definition 1.1. A subset $A$ of a topological space $(X, \tau)$ is said to be:
(1) Pre-open set [10], if $\mathrm{A} \subseteq \operatorname{Int}(\mathrm{Cl}(\mathrm{A}))$ and a pre-closed set, if $\mathrm{Cl}(\operatorname{Int}(\mathrm{A})) \subseteq \mathrm{A}$.

(2) Semi-open set [9], if $\mathrm{A} \subseteq \mathrm{Cl}(\operatorname{Int}(\mathrm{A}))$ and a semi-closed set, if $\operatorname{Int}(\mathrm{Cl}(\mathrm{A})) \subseteq \mathrm{A}$.

(3) $\alpha$-open set [13], if $\mathrm{A} \subseteq \operatorname{Int}(\mathrm{Cl}(\operatorname{Int}(\mathrm{A})))$ and a $\alpha$-closed set, if $\mathrm{Cl}(\operatorname{Int}(\mathrm{Cl}(\mathrm{A}))) \subseteq \mathrm{A}$.

(4) $\beta$-open set [1], if $\mathrm{A} \subseteq \mathrm{Cl}(\operatorname{Int}(\mathrm{Cl}(\mathrm{A})))$ and a $\beta$-closed set, if $\operatorname{Int}(\mathrm{Cl}(\operatorname{Int}(\mathrm{A}))) \subseteq \mathrm{A}$

Definition 1.2. A subset $A$ of a topological space $(X, \tau)$ is said to be:

(1) Dense set [8], if $\mathrm{Cl}(\mathrm{A})=\mathrm{X}$.

(2) Nowhere dense set [6], if Int $\mathrm{Cl}(\mathrm{A})=\varnothing$.

\section{GENERALIZED OPEN SETS WITH RESPECT TO AN IDEAL}

In this section we will discuss I- $\alpha$ (resp., I- pre, I-semi, I- $\beta$ )open sets in the topological space.

Definition 2.1. A subset $A$ of $X$ is said to be:

(1) $\alpha$-open with respect to an ideal I (written as I - $\alpha$-open), if there exists an open set $\mathrm{U}$ such that $(\mathrm{A}-\mathrm{Int} \mathrm{Cl}(\mathrm{U})) \in \mathrm{I}$ and $(\mathrm{U}-\mathrm{A}) \in \mathrm{I}$.

(2) Pre-open with respect to an ideal I (written as I-pre-open) [14], if there exists an open set $U$ such that $(A-U) \in I$ and $(\mathrm{U}-\mathrm{Cl}(\mathrm{A})) \in \mathrm{I}$.

(3) Semi-open with respect to an ideal I (written as I-semiopen) [12], if there exists an open set $U$ such that $(\mathrm{A}-\mathrm{Cl}(\mathrm{U})) \in \mathrm{I}$ and $(\mathrm{U}-\mathrm{A}) \in \mathrm{I}$.

(4) $\beta$-open with respect to an ideal I (written as I- $\beta$ - open), if there exists an open set $\mathrm{U}$ such that $(\mathrm{A}-\mathrm{Cl}(\mathrm{U})) \in \mathrm{I}$ and $(\mathrm{U}-\mathrm{Cl}(\mathrm{A})) \in \mathrm{I}$.

(5) $\alpha$ (resp., pre, semi, $\beta$ )-closed set with respect to an ideal [briefly, I- $\alpha$ (resp., I-pre, I-semi, I- $\beta$ )-closed] set iff the complement (X - A) is I- $\alpha$ (resp., I-pre, I-semi, I- $\beta$ )-open set.

Definition 2.2.If a subset A of $X$ is both I- $\alpha$ (resp., I-pre, Isemi, I- $\beta$ )-open set and I- $\alpha$ (resp., I-pre, I-semi, I- $\beta$ )-closed set, then it is called I- $\alpha$ (resp., I-pre, I-semi, I- $\beta$ )-clopen set. 
$\underline{\text { Lemma 2.3. }}$.[12] If $A \in I$, then $A$ is I -semi-open set.

\section{Remark 2.4. [11]

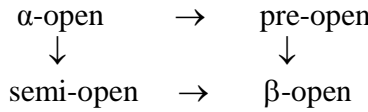

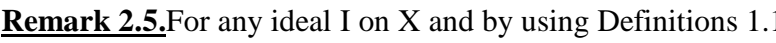
and 2.1., the following hold:

$\begin{array}{lll}\alpha \text {-open } & \rightarrow & \text { I- } \alpha \text {-open } \\ \text { semi-open } & \rightarrow & \text { I-semi-open } \\ \text { pre-open } & \rightarrow & \text { I- pre-open } \\ \beta \text {-open } & \rightarrow & \text { I- } \beta \text {-open }\end{array}$

The following examples show that the reverse implications are not satisfied.

Example 2.6 Consider a topological space $(X, \tau) ; X=\{a, b, c\}$ and $\tau=\{\varnothing,\{c\},\{a, c\}, X\}$. Choose $I=\{\varnothing,\{b\},\{c\},\{b, c\}\}$ and observe that:

(1) $\{b\}$ is I-pre ( resp., I-semi) -open; however, it is not pre(resp., semi)-open set.

(2) $\{a\}$ is I- $\alpha$ ( resp., I- $\beta$ )-open; however, it is not $\alpha$ (resp., $\beta$ ) -open set.

Lemma 2.7.For any ideal I on X, the following hold:

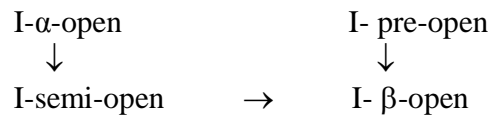

Proof In view of Definition 2.1., the proof is obvious.

Example2.8.Consider the topological space $(X, \tau)$; in example 2.6 with $\mathrm{I}=\{\varnothing,\{\mathrm{c}\}\}$. Then, $\{\mathrm{b}\}$ is I- $\alpha$-open; and is not I-preopen.

Example 2.9. Consider a topological space $(X, \tau) ; X=$ $\{a, b, c, d\}, \tau=\{\varnothing, X,\{a, b, c\},\{a, c, d\},\{a, c\}\}$ and $I=\{\varnothing$, $\{d\}\}$. Then:

(1) $\{a\}$ is I-pre-open; however, it is not I- $\alpha$-open set.

(2) $\{a\}$ is I- $\beta$-open; however, it is not I- semi-open set.

Example 2.10. Consider a topological space $(\mathrm{X}, \tau) ; \mathrm{X}=$ $\{a, b, c\}, \tau=\{\varnothing,\{b\},\{c\},\{b, c\}, X\}$ and $I=\{\varnothing,\{c\}\}$. Then:

(1) $\{a\}$ is I-semi-open; however, it is not I- $\alpha$-open set.

(2) $\{a, c\}$ is $I-\beta$-open; however, it is not I- pre-open set.

According to examples 2.8 and (i) in 2.9 the concepts of I- $\alpha$ open set and I-pre-open set are independent although every $\alpha$ open set is pre-open set in [11].

Lemma 2.11.(see [4]) If $A$ is a dense subset of $(X, \tau)$, then it is pre-open set, moreover it is $\beta$-open set.
Corollary 2.12.If $A$ is a dense subset of $(X, \tau)$, then it is Ipre-open set and I- $\beta$-open set.

Proof In view of Remark 2.5., Lemma 2.7 and Lemma 2.11, the desired proof is clear.

Lemma 2.13.If $A$ is a nowhere dense subset of $(X, \tau)$, then it is $\alpha$-closed set and semi-closed set.

Corollary 2.14 If $\mathrm{A}$ is a nowhere dense subset of $(\mathrm{X}, \tau)$ and $\mathrm{B} \subseteq \mathrm{A}$. Then,

(1) A is I- $\alpha$-closed set and I-semi-closed set.

(2) B is I- $\alpha$-closed set and I-semi-closed set.

(3) $\mathrm{Cl}(\mathrm{A})$ is I- $\alpha$-closed set and I-semi-closed set

Remark 2.15. Notice that $\tau$-open set $U$ is necessarily nonempty, otherwise for any I- $\alpha$ (resp., I-pre, I- $\beta$ ) -open subset A of $X$ and $U=\varnothing$, then $A$ is I-semi-open set.

In the next part, we discuss some of the properties of I- $\alpha$ (resp., I-pre, I-semi, I- $\beta$ )-open sets.

Proposition 2.16. $[12,14]$ If A and B are both I-semi (resp., Ipre)-open set, then so is their union $A \cup B$.

Theorem 2.17. The union of two I- $\alpha$ (resp., I- $\beta$ )-open sets of $(\mathrm{X}, \tau)$ is also an I- $\alpha$ (resp., I- $\beta$ )-open set.

Proof Let $A$ and $B$ be $I-\alpha$-open sets, then $\exists U, V \in \tau$ such that $(\mathrm{A}$ - Int $\mathrm{Cl}(\mathrm{U})) \in \mathrm{I}, \quad(\mathrm{U}-\mathrm{A}) \in \mathrm{I},(\mathrm{B}-\mathrm{Int} \mathrm{Cl}(\mathrm{V})) \in \mathrm{I}$ and $(\mathrm{V}-\mathrm{B}) \in \mathrm{I}$. Choose $\mathrm{W}=\mathrm{U} \cup \mathrm{V}$, hence $(\mathrm{U}-(\mathrm{A} \cup \mathrm{B})) \subseteq(\mathrm{U}-\mathrm{A}) \in \mathrm{I}$, $(\mathrm{V}-(\mathrm{A} \cup \mathrm{B})) \subseteq(\mathrm{V}-\mathrm{A}) \in \mathrm{I}$ and so, $(\mathrm{U}-(\mathrm{A} \cup \mathrm{B})) \cup(\mathrm{V}-(\mathrm{A} \cup \mathrm{B})) \in \mathrm{I}$. Consequently, $(\mathrm{W}-(\mathrm{A} \cup \mathrm{B})) \in \mathrm{I}$. Also, $(\mathrm{A}-\mathrm{Int} \mathrm{Cl}(\mathrm{W})) \subseteq$ $(\mathrm{A}-\mathrm{Int} \mathrm{Cl}(\mathrm{U})) \in \mathrm{I}$ and $(\mathrm{B}-\mathrm{Int} \mathrm{Cl}(\mathrm{W})) \subseteq(\mathrm{B}-\operatorname{Int} \mathrm{Cl}(\mathrm{V})) \in \mathrm{I}$ Then, $(\mathrm{A}-\mathrm{Int} \mathrm{Cl}(\mathrm{W})) \cup(\mathrm{B}-\mathrm{Int} \mathrm{Cl}(\mathrm{W})) \in \mathrm{I}$ and so $(\mathrm{A} \cup \mathrm{B}-\operatorname{Int} \mathrm{Cl}(\mathrm{W})) \subseteq(\mathrm{A}-\operatorname{Int} \mathrm{Cl}(\mathrm{U})) \cup(\mathrm{B}-\operatorname{Int} \mathrm{Cl}(\mathrm{V})) \in \mathrm{I}$. Thus $A \cup B$ is $I-\alpha$-open set. The rest of the proof is similar.

The intersection of two I- $\alpha$ (resp., I-pre, I-semi, I- $\beta$ )-open sets need not to be an I- $\alpha$ (resp., I-pre, I-semi, I- $\beta$ )-open set as shown by the following example.

Example 2.18. Consider a topological space $(X, \tau) ; X=\{a, b, c$ $\mathrm{d}\}$ and $\tau=\{\varnothing,\{a, b, d\},\{a, d\},\{a, b\},\{b, d\},\{d\},\{b\},\{a\}$, $\mathrm{X}\}$. Choose $\mathrm{I}=\{\varnothing,\{\mathrm{c}\}\}$.

(1) If $A=\{a, c\}, B=\{b, c\}$, then $A$ and $B$ are I- $\alpha$-open sets but their intersection $A \cap B=\{c\}$ is not an I- $\alpha$-open set.

(2) If $A=\{a, c\}, B=\{b, c\}$, then $A$ and $B$ are I-semi-open sets but their intersection $A \cap B=\{c\}$ is not an I-semi-open set.

(3) If $\mathrm{A}=\{\mathrm{a}, \mathrm{c}\}, \mathrm{B}=\{\mathrm{c}, \mathrm{d}\}$, then $\mathrm{A}$ and $\mathrm{B}$ are I-pre-open sets but their intersection $A \cap B=\{c\}$ is not an I-pre-open set.

(4) If $A=\{a, c\}, B=\{b, c\}$, then $A$ and $B$ are I- $\beta$-open sets but their intersection $A \cap B=\{c\}$ is not an I- $\beta$-open set.

Theorem 2.19 (1) The intersection of I-pre (resp., I-semi, I$\alpha, \mathrm{I}-\beta)$-open and open sets is an I-pre (resp., I-semi, I- $\alpha, \mathrm{I}-\beta$ )open set. 
(2) The intersection of dense and open sets is an I-pre-open set. Moreover, it is an I- $\beta$-open set.

Proof (1) Let A be an I-pre-open set and B be an open set, then there exists an open set $U$ such that $(A-U) \in I$ and $(\mathrm{U}-\mathrm{Cl}(\mathrm{A})) \in \mathrm{I}$. Since $(\mathrm{U} \cap \mathrm{B})$ is an open set, then $(A \cap B)-(U \cap B)=(A \cap B)-U \subseteq(A-U) \in I$. Since $B$ is open, then $\mathrm{Cl}(\mathrm{A}) \cap \mathrm{B} \subseteq \mathrm{Cl}(\mathrm{A} \cap \mathrm{B})$ and $((\mathrm{U} \cap \mathrm{B})-\mathrm{Cl}(\mathrm{A})) \in \mathrm{I}$. Hence, $(\mathrm{U} \cap \mathrm{B})-\mathrm{Cl}(\mathrm{A} \cap \mathrm{B}) \subseteq((\mathrm{U} \cap \mathrm{B})-\mathrm{Cl}(\mathrm{A}) \cap \mathrm{B}) \subseteq((\mathrm{U} \cap \mathrm{B})-$

$\mathrm{Cl}(\mathrm{A})) \in \mathrm{I}$. Consequently, $(\mathrm{A} \cap \mathrm{B})$ is an I-pre-open set. We can prove the rest of the theorem similarly.

(2) Let $A$ be a dense set and $B$ be an open set, then $(\mathrm{A} \cap \mathrm{B})-\mathrm{B}=\varnothing \in \mathrm{I}$. Since $\mathrm{Cl}(\mathrm{A}) \cap \mathrm{B} \subseteq \mathrm{Cl}(\mathrm{A} \cap \mathrm{B})$ and $\mathrm{Cl}(\mathrm{A})=\mathrm{X}$, then $\mathrm{B} \subseteq \mathrm{Cl}(\mathrm{A} \cap \mathrm{B})$ and so $\mathrm{B}-\mathrm{Cl}(\mathrm{A} \cap \mathrm{B})=\varnothing \in \mathrm{I}$. Consequently, $(A \cap B)$ is an I-pre-open set. By using Lemma 2.7., $(A \cap B)$ is an I- $\beta$-open set.

Theorem 2.20 If A is dense and B is open in the topological space $(\mathrm{X}, \tau)$, then $\mathrm{Cl}(\mathrm{A} \cap \mathrm{B})$ is an I-semi-open set and I- $\beta$-open set.

Proof Let $\mathrm{A}$ be a dense set and $\mathrm{B}$ be an open set, then $\mathrm{Cl}(\mathrm{A}) \cap \mathrm{B} \subseteq \mathrm{Cl}(\mathrm{A} \cap \mathrm{B})$ and $\mathrm{Cl}(\mathrm{A})=\mathrm{X}$. Hence $\mathrm{B} \subseteq \mathrm{Cl}(\mathrm{A} \cap \mathrm{B})$ and so $\mathrm{B}-\mathrm{Cl}(\mathrm{A} \cap \mathrm{B})=\varnothing \in \mathrm{I}$. Also, $\mathrm{Cl}(\mathrm{A} \cap \mathrm{B})-\mathrm{Cl} \mathrm{B}=\varnothing \in \mathrm{I}$ Consequently, $\mathrm{Cl}(\mathrm{A} \cap \mathrm{B})$ is an I-semi-open set. By using Lemma 2.7., $\mathrm{Cl}(\mathrm{A} \cap \mathrm{B})$ is an $\mathrm{I}-\beta$-open set.

The proofs of the next theorems are obvious and so are omitted.

Theorem 2.21.Let $\mathrm{I}=\{\varnothing\}$ on a topological space $\mathrm{X}$. Then: (1) The concepts of $\alpha$-openness and I- $\alpha$-openness are the same.

(2) The concepts of pre-openness and I-pre-openness are the same.

(3) The concepts of semi-openness and I-semi-openness are the same.

(4) The concepts of $\beta$-openness and I- $\beta$-openness are the same.

Theorem 2.22. Let I and $\mathrm{J}$ be two ideals on a topological space $X$. Then we have,

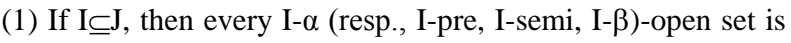
J- $\alpha$ (resp., J-pre, J-semi, J- $\beta$ )-open set.

(2) If a subset $A$ is an $(I \cap J)-\alpha$ (resp., $(I \cap J)$-pre, $(I \cap J)$-semi, $(I \cap J)-\beta$ )-open set, then it is simultaneously I- $\alpha$ (resp., I-pre, I-semi, I- $\beta$ )-open set and J- $\alpha$ (resp., J-pre, J-semi, J- $\beta$ )-open set.

Theorem 2.23. If $B$ is an I-pre (resp., I- $\beta$ )-open set and $\mathrm{A} \subseteq \mathrm{B} \subseteq \mathrm{Cl}(\mathrm{A})$ in $(\mathrm{X}, \tau)$, then $\mathrm{A}$ is an I-pre (resp., I- $\beta$ )-open set.

Corollary 2.24.Let $A$ be a subset of a topological space $X$ such that $\mathrm{Cl}(\mathrm{A})$ is an I-pre (resp., I- $\beta$ )-open set. Then $\mathrm{A}$ is an I-pre (resp., I- $\beta$ )-open set for any ideal I on X.

Corollary 2.25. Let A and B be subsets of a topological space $\mathrm{X}$ such that $\mathrm{B}$ is open, $\mathrm{A} \subseteq \mathrm{B}$, and $\mathrm{A}$ is dense in $\mathrm{B}$ (that is, $\mathrm{B} \subseteq \mathrm{Cl}(\mathrm{A})$ ). Then $\mathrm{A}$ is an I-pre-open set for any ideal I on $\mathrm{X}$.
Definition 2.26.(see [7]) A topological space $(X, \tau)$ is said to be:

(1) Hyper connected, if every nonempty open subset of $X$ is dense.

(2) Locally indiscrete, if every open subset of $X$ is closed

(3) Extremally disconnected, if the closure of every open subset of $\mathrm{X}$ is open.

Theorem 2.27.Let I be an ideal on a hyper connected topological space $(\mathrm{X}, \tau), \mathrm{A} \subseteq \mathrm{X}$ and the collection of open subsets of $\mathrm{X}$ satisfies the finite intersection property( see [8]). Then the following statements hold:

(1) If A is I-pre-closed set, then A is dense.

(2) If A is I-pre-closed set, then it is I-pre-open set.

(3) If $A$ is I-pre-closed set and $A \subseteq B$, then $B$ is I-pre-open set.

(4) If A is I-pre-closed set. Then, $A \cup B$ is I-pre-open, for any subset B of X.

(5) If $\mathrm{A}$ is I-pre-open and $\mathrm{B} \subseteq \mathrm{A}$. Then, $\mathrm{A} \cap \mathrm{B}$ is I-pre-closed, for any subset $B$ of $X$.

Proof (1) Let A be an I-pre-closed subset of X, then there exists closed set F such that (Int (A) - F) $\in \mathrm{I}$ and $(\mathrm{F}-\mathrm{A}) \in \mathrm{I}$. Since $\mathrm{X}$ is hyper connected, the collection of open subsets of $\mathrm{X}$ satisfies the finite intersection property and (Int (A) - F) is non-empty open set, then (Int (A) - F) is dense and $\mathrm{X}=$ $\mathrm{Cl}$ (Int (A) - F) $\subseteq \mathrm{Cl}$ (A) and so $\mathrm{A}$ is dense.

(2) In view of Lemma 2.7 and by using (1) of this theorem. Since A is an I-pre-closed set, then it is I-pre-open.

(3) Let $\mathrm{A}$ be I-pre-closed and $\mathrm{A} \subseteq \mathrm{B}$ and by using (1) of this theorem, then $\mathrm{A}$ is dense and so $\mathrm{B}$ is dense. In view of Lemma $2.7 \mathrm{~B}$ is I-pre-open set. The rest of the proofs are obvious.

Theorem 2.28. Let I be an ideal on a locally indiscrete topological space $(\mathrm{X}, \tau)$, then the following statements are hold:

(1) Every I-semi-open is an I-pre-open.

(2) Every I-semi-open is an I- $\alpha$-open.

(3) Every I- $\beta$-open is an I-pre-open.

(4) Every I- $\alpha$-open is an I-pre-open.

(5) If $A \subseteq X$, then $A$ is an I-pre-closed.

Proof We shall prove (1), (5) only and the rest of the proof is obvious.

(1) Let A be an I-semi-open, then there exists an open set U such that $(\mathrm{A}-\mathrm{Cl}(\mathrm{U})) \in \mathrm{I}$ and $(\mathrm{U}-\mathrm{A}) \in \mathrm{I}$. Since $\mathrm{X}$ is locally indiscrete, then $\mathrm{A}$ is an I-pre-open set.

(4) Let $A \subseteq X$. Choose $F=I n t(A)$. Since $X$ is locally indiscrete, then $\mathrm{F}$ is closed, $(\mathrm{Int}(\mathrm{A})-\mathrm{F})=\varnothing \in \mathrm{I}$ and $(\mathrm{F}-\mathrm{A})=\varnothing \in \mathrm{I}$. Consequently, $\mathrm{A}$ is an I-pre-closed set. 
Corollary 2.29. Let I be an ideal on a locally indiscrete topological space $(X, \tau)$, then the following statements are hold:

(1) Every singleton in X is I-pre-closed set.

(2) Every open subset of X is I-pre-closed set.

(3) Every open subset of X is I-pre-clopen set.

(4) Every I-semi-open set is I-pre-clopen set.

Theorem 2.30.Let I be an ideal on Extremally disconnected topological space $(\mathrm{X}, \tau)$, then the concepts I-semi-open and I- $\alpha$-open are coincide.

\section{Proof Obvious.}

\section{CONCLUSIONS}

Throughout the current paper, the notations of $\alpha$ (resp., semi, pre, $\beta$ )-open sets in terms of an ideal I have been introduced. The new concepts were found to be generalization of the usual concepts of $\alpha$ (resp., semi, pre, $\beta$ )-open sets. Several of their topological properties of these concepts were investigated.

One of them, the concepts of I- $\alpha$-open set and I-pre-open set are independent; although every $\alpha$-open set is pre-open set.

If $X$ is the locally indiscrete topological space, then every I- $\alpha$ open is an I-pre-open. Relationships were established by the new definitions of I-semi-open and I- pre-open, I- $\alpha$ open and I- $\beta$-open sets. The new findings in this paper will enhance and promote the further study on generalized open sets in terms of an ideal I to carry out a general framework for their applications in practical life.

\section{ACKNOWLEDGEMENT}

The authors are grateful to the anonymous referee for a careful checking of the details and for helpful comments that improved this paper.

\section{REFERENCES}

[1] Abd El-Monsef, M..E., El-Deeb, S. N., and Mahmould, R. A. 1983. $\beta$-open sets and $\beta$-continuous mapping. Bull. Fac. Sci. Assiut Univ., 12, 77-90.

[2] Andrijevic, D. 1986. Semi-preopen sets, Mat. Vesnik, $38,24-32$.

[3] Hamlett, T. R., and Jankovic, D. 1992. Compatible extensions of ideals, Boll. Un. Mat. Ita., 7, 453 - 465.

[4] Jafari, S., and Rajesh, N. 2011. Generalized closed sets with respect to ideals, Eur. J. Pure Appl. Math, 4(2), 147 $-151$.

[5] Jankovic, D., and Hamlett, T. R. 1990. New topologies from old via ideals, Amer. Math. Month., 97, 295 - 310.

[6] Jankovic, D., and Reilly, I. 1985. On semi-separation properties, Indian J. Pure Appl. Math. 16, 957 - 964.

[7] Julian Dontchev, 1998. Survey on pre-open sets. The Proceedings of the Yatsushiro topological conference, 118

[8] Kelley, J. L., 1955. General Topology, D.Van Nastrand Company, Inc.

[9] Levine, N., 1963. Semi-open sets and semi-continuity in topological spaces. Amer. Math. Monthly 70, 36 - 41.

[10] Mashhour, A. S., Abd El-Monsef, M. E., and El-Deeb, S. N., 1982. On pre-continuous and weak precontinuous mappings, Proc. Math. Phys. Soc. Egypt 53, 47 - 53.

[11] Mashhour, A. S., Abd El-Monsef, M. E., and Hasanein, I.A., 1984. On pretopological spaces, Bull. Math. R.S. Roumanie (N.S) 28(76), 39 - 45.

[12] Michael, F., 2013. On semi-open sets with respect to an ideal, Eur. J. Pure Appl. Math, 6, 53 - 58.

[13] Njastad, O., 1965. On some classes of nearly open sets, Pacific J. Math 15, 961 -970.

[14] Rodyna A. Hosny, 2013. Pre-open sets with ideal, European Journal of Scientific Research, 104 (1), 99 101. 